\title{
Ultra-high efficiency multilayer blazed gratings through deposition kinetic control
}

\author{
D.L. Voronov, ${ }^{*}$ E.H. Anderson, E.M. Gullikson, F. Salmassi, T.Warwick, V.V. Yashchuk, and \\ H.A. Padmore \\ Lawrence Berkeley National Laboratory, 1 Cyclotron Road, Berkeley, CA 94720 \\ *Corresponding author: dlvoronov@lbl.gov \\ Received Month X, XXXX; revised Month X, XXXX; accepted Month X, XXXX; posted Month X, XXXX (Doc. ID XXXXX); \\ published Month $X, X X X X$

\begin{abstract}
Diffraction efficiency of multilayer coated blazed gratings (MBG) strongly depends on the perfection of the saw-toothshaped layers in the overall composite structure. Growth of multilayers on saw-tooth substrates should be carefully optimized in order to reduce groove profile distortion and at the same time to avoid significant roughening of multilayer interfaces. In this work we report on a new way to optimize growth of sputter-deposited $\mathrm{Mo} / \mathrm{Si}$ multilayers on saw-tooth substrates through variation of the sputtering gas pressure. A new record for diffraction efficiency of $44 \%$ was achieved for a optimized MBG with groove density of 5250 lines $/ \mathrm{mm}$ at the wavelength of $13.1 \mathrm{~nm}$. (C) 2010 Optical Society of America

OSIS codes: 050.1950, 120.6660, 340.7480, 230.4170, 310.1860
\end{abstract}

High efficiency diffraction gratings with high groove density are of great importance for a wide range of extreme ultraviolet (EUV) and soft x-ray applications including EUV lithography [1], astrophysics and solar science [2], and high-resolution Resonance Inelastic X-ray Scattering [3]. Multilayer-coated Blazed Gratings (MBG) can offer vastly improved performance over conventional single layer-coated gratings provided that the grating fabrication process can ensure coherent addition of each layer in the multilayer stack. The main technological challenges are fabrication of nano-period saw-tooth substrates with a perfect groove profile and an atomically smooth surface of blazed facets, and deposition of a multilayer $(\mathrm{ML})$ on the highly corrugated surface of the substrates. Recent progress in fabrication of saw-tooth gratings with anisotropic etching of silicon single crystals $[4,5]$ provided the necessary quality of the substrates [6]. The main remaining problem however is coherent replication of this structure from the substrate throughout the whole multilayer stack.

Simulation of the diffraction efficiency shows that smoothening of the saw-tooth substrate by a ML deposited on it is a main limiting factor for MBG performance. Traditional deposition techniques optimized for growth of the MLs on flat substrates aim to provide interfaces of a ML stack as smooth as possible via promotion of surface relaxation processes. However, this approach does not work well for the highly corrugated surface of saw-tooth substrates, because a triangular shaped groove suffers greatly from excessive diffusion mobility of deposited atoms, caused in particular by bombardment of growing surface with energetic particles. The mobility provides effective smoothening of random roughness of interfaces, but in the same manner affects the high-frequency harmonics of a Fourier spectrum of a saw-tooth surface, causing degradation of the initial groove profile in the course the ML deposition $[7,8]$. As a result of this, a MBG loses its blazing ability and diffraction efficiency can be substantially reduced compared to the theoretical maximum.
To avoid groove degradation one should reduce the adatom mobility. However, too low a surface mobility would result in rough interfaces and increase the risk of shadowing effects. The shadowing can trigger a columnar growth regime which would ruin the periodicity of a ML stack and result in dramatic degradation of $\mathrm{ML}$ reflectance [9]. Since shadowing increases with the angle of deposition it is extremely problematic for saw-tooth substrates which have significant local slope variations.

The traditional strategy for deposition should be reconsidered in terms of saw-tooth substrate demands and a deposition process should be optimized in order to find a balance between roughening and smoothening, specifically for MBG purposes. The surface mobility of the adatoms should be tuned carefully in order to provide good replication of the groove profile by a ML stack, and at the same time to avoid excessive roughening of the $\mathrm{ML}$ interfaces and shadowing effects in order to keep ML reflectance high.

Surface relaxation processes can be controlled by the temperature of a substrate or the energy of deposited particles. Low-temperature deposition was found to reduce intermixing of Mo and Si layers, but resulted in very rough interfaces [10]. Control of adatom mobility by variation of sputtering gas pressure [9,11], which affects the energy of deposited particles, seems the most promising route to kinetic control. In this case an optimal pressure of a sputtering gas should be found for a particular multilayer material since the adatom mobility depends also on the surface properties of a material. Here we report on the first results of such kind of optimization for growth of dc-magnetron sputtered Mo/Si MLs on sawtooth substrates with a blaze angle of $2^{\circ}$ and a groove density of 5250 lines/mm.

We fabricated saw-tooth substrates with a process based on anisotropic etching of silicon $[4,5]$. Float zone silicon (111) wafers with the miscut of $2^{\circ}$ towards the $<112>$ direction were used for the substrate fabrication. The wafers were preliminary coated with a $30 \mathrm{~nm}$ thick low-stress nitride layer. Subsequently, they were spin- 
coated with UV210 photo-resist, and then patterned with a simple Lloyd's mirror interference setup similar to those mentioned in literature [12]. Briefly, a beam of a $266 \mathrm{~nm}$ c.w. laser light was focused onto a spatial filter with a diameter of $5 \mu \mathrm{m}$ to produce a spherical wave behind the pinhole. The divergent beam illuminated simultaneously the wafer and the Lloyd's mirror attached to the wafer at an angle of $90^{\circ}$. Fringes were formed on the wafer surface as a result of interference of the direct wave and the one reflected from the Lloyd's mirror. The incidence angle on the Lloyd's mirror was $44.43^{\circ}$ and was chosen to obtain a fringe period of $190 \mathrm{~nm}$.

After the exposure, resist bake and development, the pattern was transferred to the nitride layer with a reactive ion etch to form a hard mask. Anisotropic etching of the silicon followed by isotropic chemical etch was used to produce triangular grooves in the saw-tooth substrates with the blaze angle of $2^{\circ}$. The details of the etch process can be found elsewhere [6].

For a grating period of $190 \mathrm{~nm}$ and a blaze angle of $2^{\circ}$, the depth of the triangular grooves in the direction perpendicular to the blazed facets is $190 \times \sin 2^{\circ}=6.63 \mathrm{~nm}$ which matches to the bi-layer thickness of the EUV Mo/Si multilayer. This provides a blaze condition for the $1^{\text {st }}$ diffraction order of the MBG.

In order to optimize the Ar pressure a few Mo/Si MLs were deposited on flat substrates over a wide range of the pressures. Atomic force microscope (AFM) measurements of the top surface of the MLs over $1 \times 1 \mu \mathrm{m}^{2}$ areas showed that increase of the pressure from $1 \mathrm{~m}$ Torr to $5 \mathrm{~m}$ Torr resulted in surface roughness built-up from $0.12 \mathrm{~nm}$ up to $0.5 \mathrm{~nm} \mathrm{rms}$ and significant reduction of the $\mathrm{ML}$ reflectance. The pressure of $3.5 \mathrm{mTorr}$ was found to be optimal since a reasonable roughness of $0.23 \mathrm{~nm}$ could be achieved and did not significantly affect the $\mathrm{ML}$ performance. The details of the optimization will be published elsewhere.

Two Mo/Si MLs consisting of 30 bi-layers with a bi-layer thickness of $6.7 \mathrm{~nm}$ were deposited on saw-tooth substrates at different pressures of Ar gas. The grating \#1 was coated at an Ar gas pressure of $1 \mathrm{~m}$ Torr. At this pressure a free mean path of particles approximately corresponds to the target-substrate distance of $75 \mathrm{~mm}$. Under these conditions a growing surface undergoes extensive bombardment with energetic particles. The surface relaxation processes stimulated by the bombardment ensure effective suppression of surface roughness and results in smooth interfaces of the $\mathrm{ML}$ stack and highest performance of a ML mirror. Grating \#2 was deposited at an Ar pressure of 3.5 mTorr. In this case the free mean path is much smaller than the targetsubstrate distance, therefore particles arriving at the substrate are expected to be reasonably well thermalized. Under these conditions the adatom mobility should be limited, and the $\mathrm{ML}$ is expected to have better replication performance. Under these conditions, since the ability of the multilayer to smooth high-frequency surface features is reduced, shadowing effects can result in a very undesirable columnar growth regime. In order to prevent this possible shadowing in the very beginning of the deposition an additional buffer layer of silicon with the thickness of $20 \mathrm{~nm}$ was deposited at a pressure of $1 \mathrm{mTorr}$ prior to the $\mathrm{ML}$ deposition. The layer was designed to provide a minimal smoothening of the substrate to reduce the risk of shadowing especially in the vicinity of highly tilted surface areas of anti-blazed facets of the saw-tooth substrate. Two witness $\mathrm{Mo} / \mathrm{Si}-30 \mathrm{MLs}$ were deposited on flat silicon substrates at an $\mathrm{Ar}$ pressure of 1 and 3.5 mTorr simultaneously with the respective MBGs in order to monitor the $\mathrm{ML}$ reflectance depending on the pressure.

Cross-section TEM images of the gratings are shown in Fig. 1. As expected, the low-pressure deposition results in smooth interfaces of the ML stack, but at the same time the grooves are substantially smoothed (Fig. 1a). In contrast to the low-pressure deposition, the consequences of reduced adatom mobility are clearly visible for the higher pressure deposition. The roughness of the interfaces gradually increases from the bottom to the top of the ML stack (Fig. 1b). The roughening of the interfaces is consistent with the top surface roughness built-up observed with AFM for high pressure deposition. As desired however, the ML with reduced smoothing ability replicates the substrate relief much better, and the sawtooth groove profile propagates through the whole $\mathrm{ML}$ stack with almost no distortion.
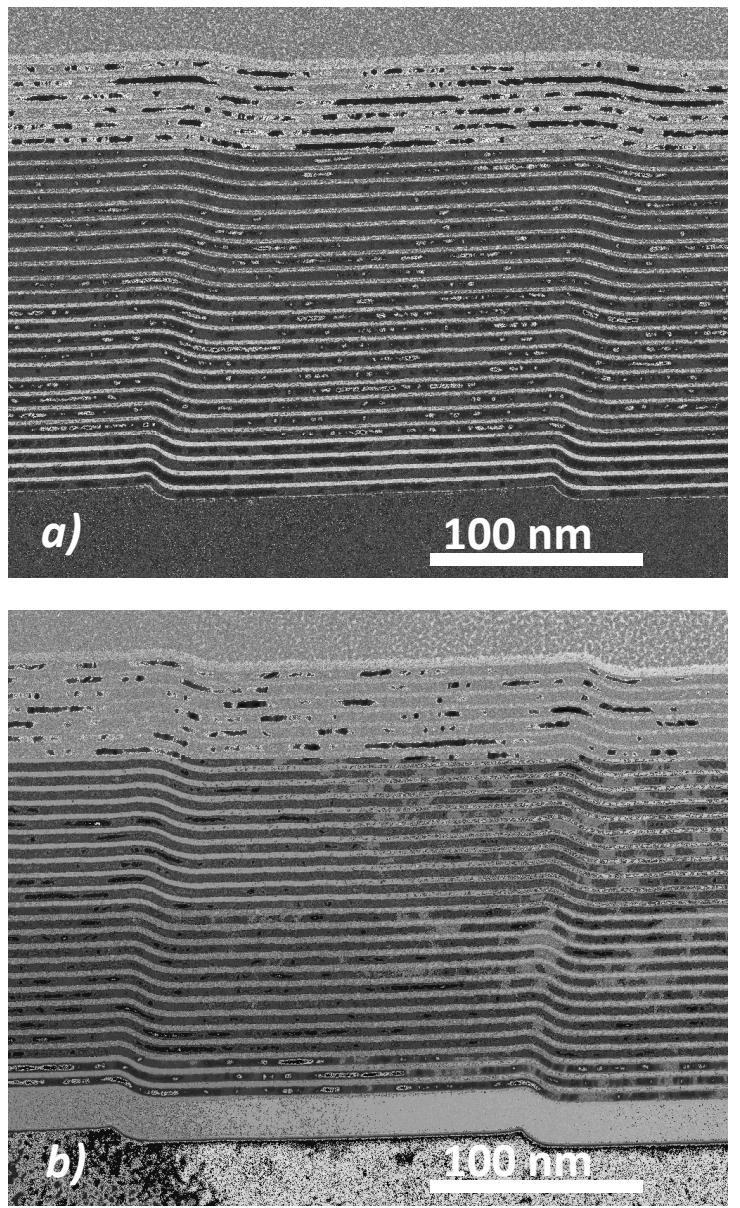

Fig. 1. Cross-section TEM images of gratings \#1 (a) and \#2 (b) coated with $\mathrm{Mo} / \mathrm{Si}-30$ multilayers, deposited at an $\mathrm{Ar}$ gas pressure of $1 \mathrm{~m}$ Torr and $3.5 \mathrm{~m}$ Torr respectively. Increase of the pressure results in some roughening of interfaces, but provides much better replication of the saw-tooth grooves by the ML. 
Measurements of diffraction efficiency of the MBGs shown in Fig. 2 were performed at a constant angle of incidence of $7^{\circ}$ from the grating normal by detector scan over diffraction angles at the $\mathrm{ML}$ resonance wavelength. The diffraction patterns consist of a strong peak of the $1^{\text {st }}$ blazed order and minor peaks of non-blazed orders. Reflectance of the witness Mo/Si-30 MLs was measured over EUV wavelength range at a constant Bragg's angle of $85^{\circ}$. The reflectance curves are plotted in incretions in Fig. 2. The roughening of the interfaces observed for the MBG \#2 causes some minor reduction of reflectance of the $\mathrm{ML}$ witnesses from $63.7 \%$ to $62.2 \%$ for 1 and 3.5 mTorr depositions respectively. Such a moderate reduction of the ML reflectance does not significantly affect the performance of the MBG \#2. At the same time the grating has much better blazing ability due to better replication of the groove profile. The improved blazing of the grating \#2 provides stronger suppression of the non-blazed diffraction orders as compared to the grating \#1 (see Fig. 2a and 2b), while more diffracted energy is concentrated into the $1^{\text {st }}$ blazed order. As a result of this, the efficiency of the blazed order increases from $39.6 \%$ for grating \#1 to $44.0 \%$ for grating \#2.
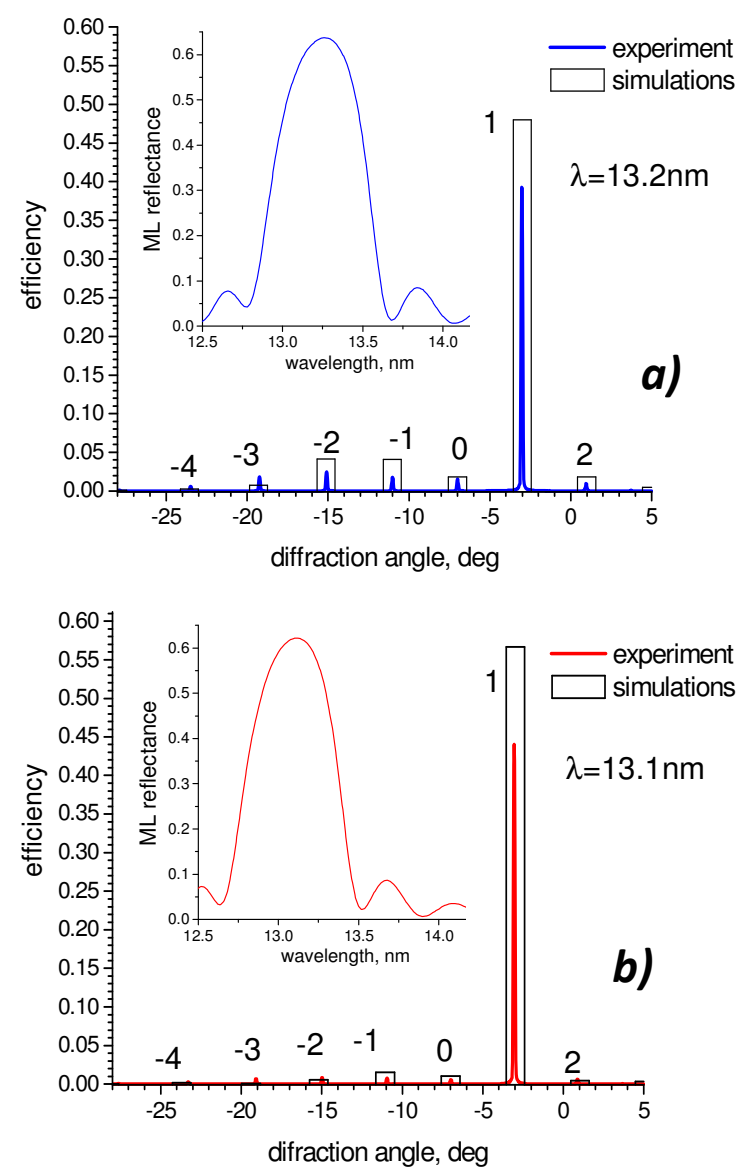

Fig. 2 (Color online). Diffraction from the gratings \#1 (a) and \#2 (b) coated with $\mathrm{Mo} / \mathrm{Si}^{-30}$ multilayers deposited at the $\mathrm{Ar}$ gas pressure of $1 \mathrm{~m}$ Torr and $3.5 \mathrm{~m}$ Torr respectively. Reflectance of the witness $\mathrm{Mo} / \mathrm{Si}^{-30} \mathrm{MLs}$ versus wavelengths is shown in insertions.

The measurements of diffraction efficiency are consistent with efficiency simulations based on the integral method for solving Maxwell's equations for a periodic structure [13]. Average groove profiles obtained from AFM measurements of the top surface of the MBGs were used for groove shape modeling, while $\mathrm{ML}$ interfaces were assumed to be ideally smooth and sharp. The theoretical efficiency of $56.7 \%$ calculated using the real shape of grating \#2 is somewhat lower as compared to the efficiency of $67.6 \%$ of an ideal MBG with a perfect sawtooth profile (not shown). Calculated diffraction efficiency for grating \#1 does not exceed $48 \%$ due to the smoothed groove profile.

In summary, we showed that performance of a MBG can be significantly improved by optimization of growth condition of a ML on the highly corrugated surface of a saw-tooth substrate. The optimal balance between roughening and smoothening processes controlled by sputtering gas pressure was found experimentally. Although increase of the pressure results in some built-up of roughness of the ML interfaces, better replication of the saw-tooth grooves by the $\mathrm{ML}$ enhances the blazing ability and improves the diffraction efficiency of the MBG. As a result of the optimization an absolute efficiency of $44 \%$ was achieved for the $1^{\text {st }}$ blazed order of a $5250 \mathrm{l} / \mathrm{mm}$ MBG. To our best knowledge this is a highest efficiency has ever been achieved for dense multilayer gratings.

This work was supported by the US Department of Energy under contract number DE-AC02-05CH11231.

\section{References}

1. P.P. Naulleau, W.C. Sweatt, D.A. Tichenor, Opt. Comm., 214, 31-38 (2002).

2. M.P. Kowalski, R.G. Cruddace, K.F. Heidemann, R. Lenke, H. Kierey, T.W. Barbee Jr., and W.R. Hunter, Opt. Lett. 29, 2914-2916 (2004).

3. A. Kotani, Sh. Shin, "Resonant inelastic x-ray scattering spectra for electrons in solids," Rev. Mod. Phys. 73, 203-246 (2001).

4. Y. Fujii, K.I. Aoyama, and J.I. Minowa, IEEE J. Quantum Electron. QE-16, 165-169 (1980).

5. P. Philippe, S. Valette, O. Mata Mendez, D. Maystre, Appl. Opt. 24, 1006-1011 (1985).

6. D.L. Voronov, M. Ahn, E.H. Anderson, R. Cambie, Ch.-H. Chang, E.M. Gullikson, R.K. Heilmann, F. Salmassi, M.L. Schattenburg, T. Warwick, V.V. Yashchuk, L. Zipp, H.A. Padmore, Opt. Lett. 35, 2615-2618 (2010).

7. D.L. Voronov, M. Ahn, E.H. Anderson, R. Cambie, Ch.-H. Chang, L.I. Goray, E.M. Gullikson, R.K. Heilmann, F. Salmassi, M.L. Schattenburg, T. Warwick, V.V. Yashchuk, and H.A. Padmore, Proc. SPIE 7802, 780207 (2010).

8. D.L. Voronov, E.H. Anderson, R. Cambie, S. Cabrini, S.D. Dhuey, L.I. Goray, E.M. Gullikson, F. Salmassi, T. Warwick, V.V. Yashchuk, and H.A. Padmore, Opt. Express 19, 6320-6325 (2011).

9. D. G. Stearns, R. S. Rosen, S. P. Vernon, J. Vac. Sci. Technol. A 9, 2662-2669 (1991).

10. V.I.T.A. de RooijLohmann, A.E. Yakshin, E. Zoethout, J. Verhoeven, F. Bijkerk, Appl. Surf. Science 257, 6251-6255 (2011).

11. A.E. Yakshin, R.W.E. van de Kruijs, I. Nedelcu, E. Zoethout, E. Louis, and F. Bijkerk, Proc. of SPIE 6517, 65170I (2007).

12. E. Hecht, Optics (Addison Wesley, 2002).

13. http://www.pcgrate.com 


\section{DISCLAIMER}

This document was prepared as an account of work sponsored by the United States Government. While this document is believed to contain correct information, neither the United States Government nor any agency thereof, nor the Regents of the University of California, nor any of their employees, makes any warranty, express or implied, or assumes any legal responsibility for the accuracy, completeness, or usefulness of any information, apparatus, product, or process disclosed, or represents that its use would not infringe privately owned rights. Reference herein to any specific commercial product, process, or service by its trade name, trademark, manufacturer, or otherwise, does not necessarily constitute or imply its endorsement, recommendation, or favoring by the United States Government or any agency thereof, or the Regents of the University of California. The views and opinions of authors expressed herein do not necessarily state or reflect those of the United States Government or any agency thereof or the Regents of the University of California. 